\title{
Adaptive Filter and Resonant Controller Applied to Hybrid Series Active Power Filter
}

\author{
R.B. Gonzatti ${ }^{1}$, S.C. Ferreira ${ }^{1}$, Carlos H. da Silva ${ }^{1}$, L.E. Borges da Silva ${ }^{1}$, \\ G. Lambert-Torres ${ }^{1}$, and Se Un $\mathrm{Ahn}^{2}$ \\ ${ }^{1}$ Federal University of Itajuba - UNIFEI, Itajuba - Brazil \\ ${ }^{2}$ CPFL Energia - Piratininga - Brazil \\ \{carloschedas, leborges\} @unifei.edu.br
}

\begin{abstract}
This paper proposes a control strategy for Hybrid Active Series Power Filter (HASPF) based on a P+Resonant compensator. The proposed control uses Adaptive Notch Filter to isolate the harmonic content of the source current and a $\mathrm{P}+$ Resonant regulator to calculate the harmonic voltage signal necessary to properly tune the hybrid branch. This control strategy ensures multiple frequencies tuning by using only one passive filter. The details of the control algorithm and the design procedure are presented. Experimental results using a single-phase HASPF prototype confirm the effectiveness of the suggested approach.
\end{abstract}

Keywords: Active Power Filter, Proportional Resonant Regulator, Adaptive Notch Filter, Harmonic Mitigation.

\section{Introduction}

The Hybrid Active Series Power Filter (HASPF) is generally composed by one smallrated Active Filter (AF) associated in series with a tuned passive filter (PF) [1], [2]. This Hybrid topology, shown in Fig. 1, significantly improves the compensation characteristics of simple LC passive filters.

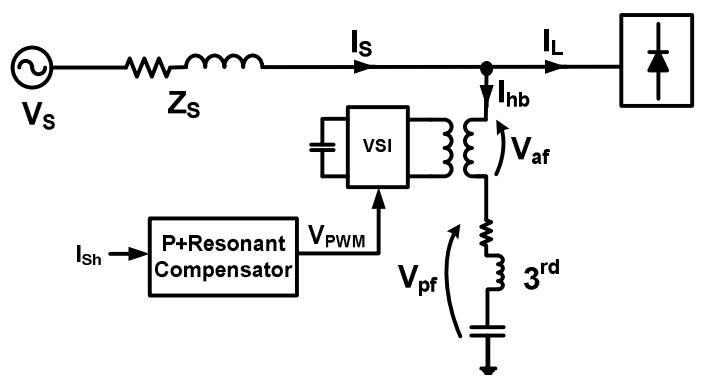

Fig. 1. Hybrid Active Series Power Filter

In order to achieve the ideal hybrid filter tuning an appropriate control strategy is crucial. Many hybrid filter control schemes have been proposed, based on the $p-q$ 
theory/Instantaneous Reactive Power Reference [1] or on Synchronous Reference Frame (SRF) [2], [3], [4].

The key strategy of the SRF controller is that the current component at the same frequency of the SRF becomes a DC signal that can be isolated with a low-pass filter (LPF). As long as the desired harmonic component becomes a DC signal, a conventional PI controller can achieve zero steady-state error and thus eliminate that specific harmonic component. However, for each harmonic frequency to be mitigated it is necessary to implement a respective SRF. It represents a very important drawback of this approach.

In order to deal with the problems regarding sinusoidal references a Proportional Resonant ( $\mathrm{P}+\mathrm{Resonant}$ ) regulator was originally proposed for current control at fundamental frequency [5], [6]. This regulator, with appropriate tuning, has infinite gain in its resonant frequency achieving zero steady-state error at this frequency. It can be seen as having the same transient and steady-state characteristics, for a sinusoidal reference at its resonance frequency, as a PI regulator has for a continuous reference. Due to these features, resonant compensators became of interest in the area of Active Filters and have been successfully applied to harmonic current control in Shunt Active Filters (SAF) [7], [8].

In this paper the $\mathrm{P}+$ Resonant compensator is applied to voltage control of a HASPF. This strategy indirectly controls the current by controlling the output voltage imposed by the AF, which implements an active impedance providing an ideal short circuit for the harmonic load current [4]. Also, this technique allows compensating more than one frequency at the same time with only one passive filter.

In order to guarantee no influence of the controller on the fundamental source current, an Adaptive Notch Filter is used to isolate the harmonic content from the fundamental component [9],[10].

To demonstrate the effectiveness of the proposed strategy, practical results are presented for a single-phase prototype compensating simultaneously four harmonics frequencies. The setup uses a DSP TMS320F2812 for digital implementation of the Proportional Resonant controller.

\section{Contribution to Value Creation}

The value creation can be divided in two main sections. The conditioner topology which is based on the active impedance and the control strategy based on adaptive plus resonant concepts.

Referring to hybrid active power filters, the value added result from the independency of passive filter components tolerance, which is critical, and from reducing the number of tuned passive filter demanded. Only one tuned filter is needed to mitigate any harmonic current drawn by the power system.

A smaller passive filter cell is cheaper, and then it will reduce the cost associated to harmonic mitigation increasing the compensation effectiveness.

Besides the conditioner topology, the proposed controller is based on unusual harmonic detection methods and allows an active impedance implementation 
increasing the number of harmonic current components to be mitigated, adding value to the proposed active filter.

\section{Hybrid Series Active Filter Fundamentals}

Fig.1 shows the hybrid branch which combines an active filter and a passive filter. The active filter is a controllable voltage source which provides any desired voltage, at fundamental or harmonic frequencies. Considering the command of instantaneous voltage of active filter, $V_{a f}$ is given by

$$
V_{a f}=-K_{a f} \cdot V_{f p h}
$$

Here, $K_{a f}$ is a gain and $V_{f p h}$ is the harmonic voltage across the passive filter. At this situation, the specific harmonic voltage, at the hybrid branch is zero, resulting in a short-circuit in that frequency. Assuming the source voltage is free from harmonics, (2) is satisfied since $K_{a f}$ is unity, under ideal conditions.

$$
V_{s}(h)=V_{a f}(h)+V_{p f}(h)
$$

Under real conditions, the information about the harmonics flowing through the hybrid branch depends on the voltage source distortion and load current harmonics. So, (1) can be rewritten as,

$$
V_{a f}=-K_{a f}\left(V_{s h} \cdot\left(Z_{h b} / Z_{s}\right)+Z_{h b} \cdot I_{L h}\right)
$$

where $V_{s h}$ is the harmonic source voltage, $Z_{h b}$ is the equivalent passive intrinsic impedance of the transformer $\left(Z_{t}=r_{t}+j X_{t}\right)$ plus that of the passive filter $\left(Z_{p f}=r_{p f} \pm\right.$ $\left.j X_{p f}\right), Z_{s}$ is the source equivalent impedance and $I_{L h}$ is the harmonic load current.

\section{$4 \quad$ Active Impedance Principle}

Two important performance indexes must be taken in account when talking about filtering (passive or active), the tuned factor and the quality factor [4].

The quality factor can be related to hybrid filter concept as follows:

$$
Q=\frac{1}{\left(r_{t}+r_{p f}+r_{\text {active }}\right)} \sqrt{\frac{\left(L_{t}+L_{p f}+L_{a c t i v e}\right)}{C}}
$$

where $L_{t}$ is the transformer equivalent inductance, $r_{t}$ is the transformer equivalent resistance, $L_{p f}$ is the passive inductance and $r_{p f}$ is the passive filter resistance. $L_{\text {active }}$ and $r_{\text {active }}$ are respectively the real and imaginary part of extended concept of the active filter gain $K_{a f}$, referred as active impedance [4].

$$
k_{\text {af }}=-r_{\text {active }} \pm j 2 \pi f L_{\text {active }}
$$


The real and imaginary parts of the active impedance are manipulated independently by the active filter in order to adjust the hybrid filter quality and tuned factor.

It is important to notice the capability of this structure, HASPF and P+Resonant controller, to create multiple selective tuning. It opens the possibility to implement simultaneously several active impedances.

The compensation for multiples tune frequencies can be reached according to (6).

$$
\sum_{i=1}^{N} f(i)=\sum_{i=1}^{N}(\underbrace{\frac{1}{2 \pi \sqrt{\left(L_{a}(i)+L_{i}+L_{p f}\right) C}}}_{f(i)})
$$

Where $L_{a}(i)$ is the component $i$ of the active impedance which tuned the frequency $f(i) . L_{t}$ is the transformer inductance when it is not negligible.

The controller calculates the voltage needed to be applied by the AF in order to implement the active impedance $k_{a f}=-r_{f} \pm j 2 \pi f_{h} L_{a}$, thus improving the quality and tuned factor of the passive filter.

The control scheme assumes that once the harmonic current component flowing in the source is equal to zero all the harmonic current generated by the load flows through the hybrid branch. When this happens a properly tuning of the hybrid branch has been achieved.

\section{The Proportional Resonant Compensator}

The Proportional Resonant (P+Resonant) regulator has been proposed for current control at fundamental frequency in a stationary frame with a transfer function based on LC resonant circuit [5]. This compensator provides steady-state error for a sinusoidal reference because it has a high gain at its resonance frequency. Then a similar regulator has been presented [6] based on an exact mathematical transformation from the equivalent ideal synchronous frame PI, which is able to track a reference at the same frequency as the rotating frame with zero steady-state error.

The P+Resonant regulator adopted in this paper has the transfer function proposed in [6] and given by (7). It presents a better phase margin and transient performance in closed loop operation.

$$
C(s)=k_{p}+\frac{k_{r} s}{s^{2}+\omega_{0}^{2}}
$$

where $k_{p}$ is the proportional gain, $k_{r}$ is the resonant term gain and $\omega_{0}$ is the resonance frequency.

The control loop for the HASPF is shown in Fig. 2. The supply impedance is usually ignored in the design. The PWM, inverter and control hardware are assumed to be a simple gain (Ks) and a delay (Ts).

For this application the regulator resonant frequency and the passive filter tuning frequency are the same. Although, more harmonics can be mitigated, as the controller is able to track references at several frequencies simultaneously using a sum of resonant terms, each one with a different resonance frequency. 


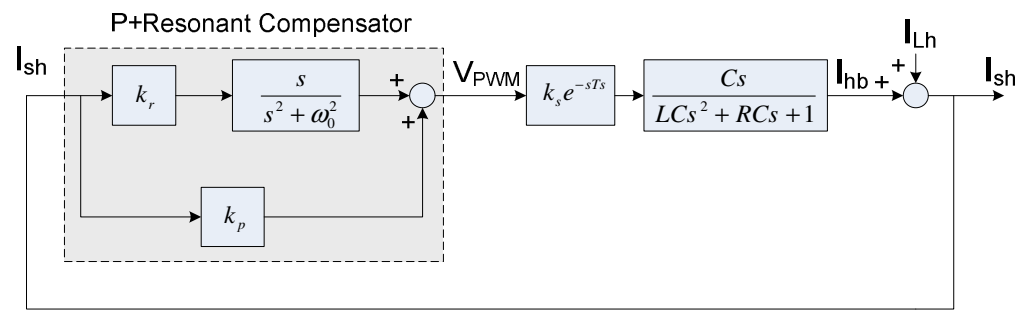

Fig. 2. Control loop scheme for the HASPF

\section{The Principle of Adaptive Notch Filter Based on LMS Algorithm}

An adaptive filter is essentially a digital filter with self-adjusting characteristics, which adapts automatically to changes in its input signal.

The structure in Fig. 3 shows an adaptive filter working as a notch filter. This filter is previously proposed on the literature for harmonic detection of current for power quality improvement [9], [10].

In this structure of adaptive filter applied to harmonic detection, $d(n)$ represents the signal with harmonics, $x(n)$ and $x_{90^{\circ}}(n)$ represents two orthogonal signals with a fundamental frequency of $60 \mathrm{~Hz}$, as reference. The reference input signals are processed by the adaptive filter producing the output signal $y(n)$ that tracks the variation in the fundamental signal. The coefficients of this filter are adjusted using the least mean square (LMS) algorithm.

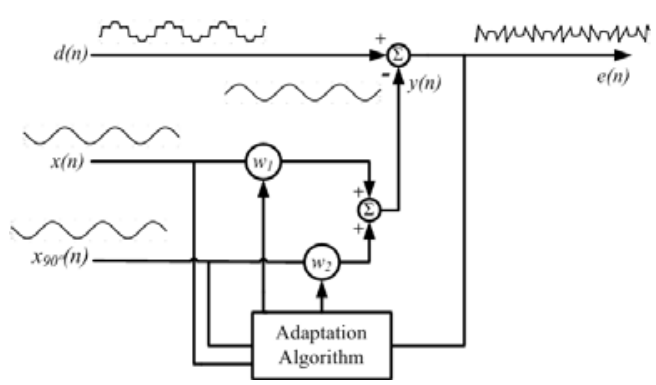

Fig. 3. Adaptive notch filter principle [10]

The general recursion formula of LMS algorithm applied for adaptive notch filters is given by:

$$
\begin{gathered}
y(n)=w_{1}(n) x(n)+w_{2}(n) x_{90^{\circ}}(n) \\
e(n)=d(n)-y(n) \\
w_{1}(n+1)=w_{1}(n)+\mu e(n) x(n)
\end{gathered}
$$




$$
w_{2}(n+1)=w_{2}(n)+\mu e(n) x_{90^{\circ}}(n)
$$

Where: $x(n), x_{90^{\circ}}(n)$ are the input signals; $w_{l}(n), w_{2}(n)$ are filter coefficients and $\mu$ is step-size value.

The parameter $\mu$ controls the algorithm rate of convergence to the optimum solution. In the adaptive notch filter only two coefficients are needed to be adapted, which makes this technique faster than the others.

As a result, this structure is used to voltage and current harmonic content detection. In this case, the reference signals are provided by a PLL (Phase Locked Loop). The PLL used is proposed by [11], and it returns a unit sine and cosine which will be used as $x(n)$ and $x_{90^{\circ}}(n)$.

\section{The Proposed Control Strategy}

The proposed control strategy is shown in Fig. 4. Firstly, the harmonic current content $I_{s h}$ is isolated from the source current $I_{s}$ using the Adaptive Notch Filter [10], in order to eliminate all possible effects on the fundamental source current. Later the harmonic current $\mathrm{I}_{\mathrm{sh}}$ is compensated by the $\mathrm{P}+\mathrm{Resonant}$ regulator that generates the appropriate voltage reference $\mathrm{V}_{\mathrm{PWM}}$ to eliminate the harmonic current from the source. Optimal quality and tuned factor is achieved for the hybrid filter on the chosen frequency.

Using one resonant term for each frequency, as in Fig. 4, several harmonics can be compensated with no need of another tuned passive filter. Each resonant term calculates the voltage necessary to create the active impedance $k_{a f}=-r_{f} \pm j 2 \pi f_{h} L_{a}$ to retune the hybrid branch to a new frequency.

The Bode plot of the P+Resonant compensator for four frequencies is also shown in Fig. 4. The peaks at the resonance frequencies can be clearly seen.
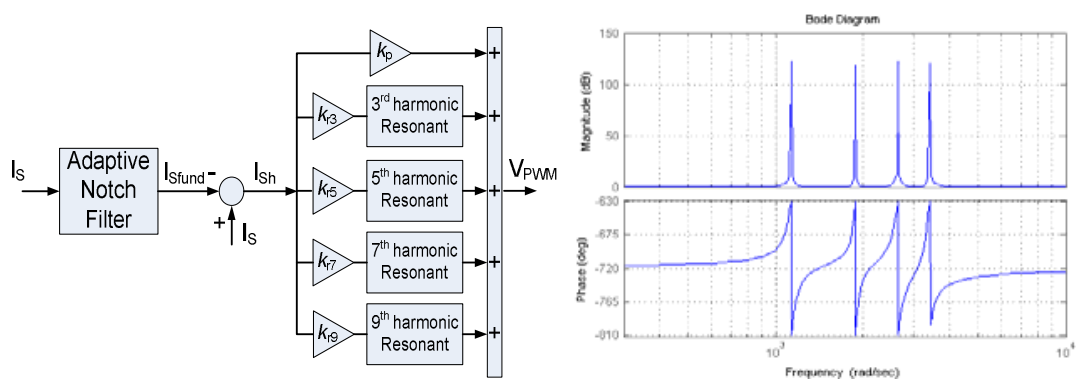

Fig. 4. Proposed Control Strategy and P+Resonant Controller Structure for Multiple Frequency Compensation and Bode plot of the compensator for multiple frequencies

\subsection{Control Design Considerations}

A method for gain specification based on the closed loop root locus analysis was used. The resonant term provides very little gain outside the band-pass region, shown in Fig. 4. Hence, to achieve a better transient response a proportional gain $k_{p}$ must be included. A large proportional gain assures a fast transient response. However, if the proportional gain is too large it can lead the system to instability. 
The proportional gain $k_{p}=10$ gives a good transient response and also ensures that all roots remain on the left of the stability boundary. A gain $k_{r}=7000$ guarantees total elimination harmonic current. All the model simulation, stability and transient responses simulation were made using the software MATLAB®/Simulink.

\section{$8 \quad$ Practical Results of Control Implementation}

To confirm the effectiveness of the suggested control strategy practical results are presented using a single-phase HASPF prototype. The system under test uses as load a full-bridge single-phase rectifier connected to a $30 \Omega$ resistance and $100 \mathrm{mH}$ inductance fed by a 127 Vrms phase-neutral voltage.

The Active Filter is composed by $\mathrm{H}$-bridge inverter connected to a $1 \mathrm{kVA}$ transformer with turn ratio of 1:4 (110/440V). The inverter switching frequency is 20 $\mathrm{kHz}$ and the DC link voltage is regulated to $440 \mathrm{Vdc}$. The Passive Filter is tuned for the $3^{\text {rd }}$ harmonic $(180 \mathrm{~Hz})$, the major harmonic current component of the load.

Fig. 5 shows the power system signals before the HASPF starts compensating and the THD of source current $I_{s}$ that is $32.7 \%$. The non-ideality of active filter coupling transformer dismisses the tune frequency of the passive filter, not mitigating the third harmonic as expected.
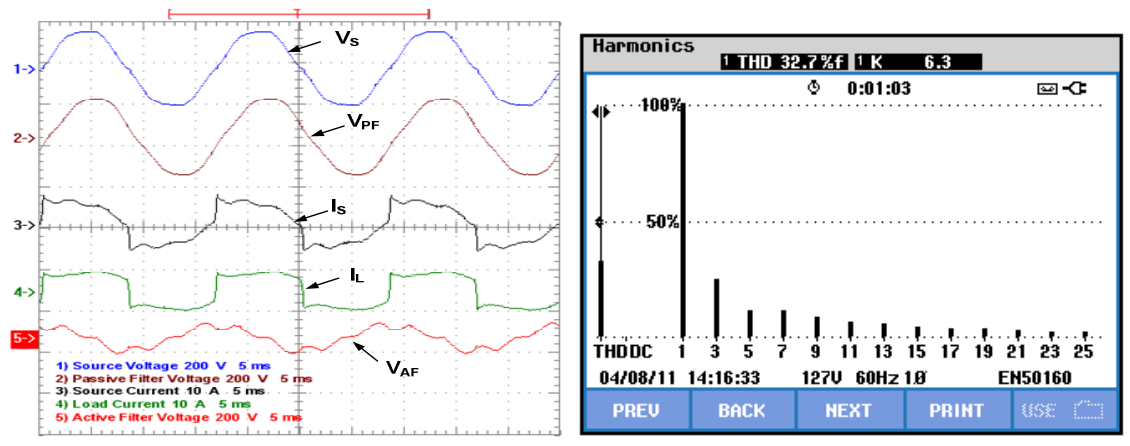

Fig. 5. System power signals and Source current spectrum before active compensation
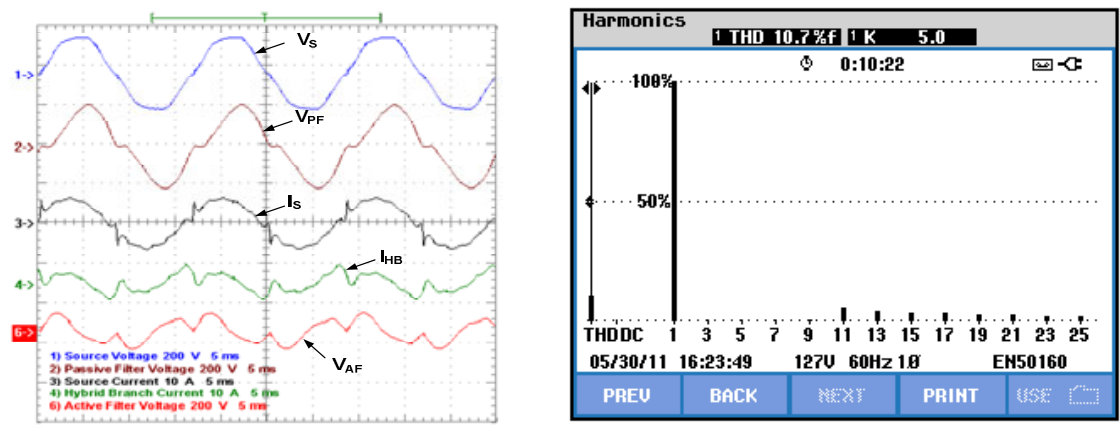

Fig. 6. System power signals and source current spectrum under 3rd, 5th, 7th and 9th active compensation 
Fig. 6 shows the power system signals and the source current spectrum with the $\mathrm{P}+$ Resonant regulator tuned for third, fifth, seventh and ninth harmonic respectively. The THD of source current $\mathrm{I}_{\mathrm{s}}$ is $10.7 \%$.

\section{Conclusions}

The developed control technique, applied to the HASPF (Hybrid Active Series Power Filter), shows an excellent performance concerning harmonic current compensation. The $\mathrm{P}+$ Resonant strategy provides accurate control of the imposed harmonic voltage, significantly enhancing the quality and tuned factor of the passive filter. Also brings improvement to the standard SRF-PI reducing DSP processing time and hence allowing multiple frequencies tuning choice.

\section{References}

1. Fujita, H., Akagi, H.: A practical approach to harmonic compensation in power systemsseries connection of passive and active filters. IEEE Transactions on Industry Applications 27(6), 1020-1025 (1991)

2. Bhattacharya, S., Divan, D.M., Banerjee, B.: Synchronous frame harmonic isolator using active series filter. In: EPE Conf. Record, vol. 3, pp. 30-35 (1991)

3. Cheng, P.-T., Bhattacharya, S., Divan, D.: Operations of the dominant harmonic active filter (DHAF) under realistic utility conditions. IEEE Transactions on Industry Applications 37(4), 1037-1044 (2001)

4. Silva, C.H., Pereira, R.R., Silva, L.E.B., Lambert-Torres, G., Pinto, J.O.P.: Modified synchronous reference frame strategy for selective-tuned single phase hybrid active power filter. In: Proceedings of the IEEE IAS 2009 (2009)

5. Sato, Y., Ishizuka, T., Nezu, K., Kataoka, T.: A new control strategy for voltage-type PWM rectifiers to realize zero steady-state control error in input current. IEEE Transactions on Industry Applications 34(3), 480-486 (1998)

6. Zmood, D.N., Holmes, D.G., Bode, G.H.: Frequency-domain analysis of three-phase linear current regulators. IEEE Transactions on Industry Applications 37(2), 601-610 (2001)

7. Lenwari, W., Sumner, M., Zanchetta, P.: Design and analysis of high performance current control for shunt active filters. In: The 3rd IET International Conference on Power Electronics, Machines and Drives, PEMD 2006, April 4-6, pp. 90-95 (2006)

8. Bojoi, R., Limongi, L.R., Roiu, D., Tenconi, A.: Frequency-domain analysis of resonant current controllers for active power conditioners. In: 34th Annual Conference of IEEE Industrial Electronics, IECON 2008, November 10-13, pp. 3141-3148 (2008)

9. Widrow, B., Glover Jr., J.R., McCool, J.M., Kaunitz, J., Williams, C.S., Hearn, R.H., Zeidler, J.R., Dong Jr., E., Goodlin, R.C.: Adaptive noise cancelling: Principles and applications. Proceedings of the IEEE 63(12), 1692-1716 (1975)

10. Pereira, R.R., da Silva, C.H., da Silva, L.E.B., Lambert-Torres, G., Pinto, J.O.P.: Improving the convergence time of adaptive notch filters to harmonic detection. In: IECON 2010-Conference on IEEE Industrial Electronics Society, November 7-10, pp. 521-525 (2010)

11. Silva, C.H., Pereira, R.R., Silva, L.E.B., Lambert-Torres, G., Bose, B.K., Ahn, S.U.: A digital PLL scheme for three-phase system using modified synchronous reference frame. IEEE Trans. on Industrial Electronics 57(11), 3814-3821 (2010) 